\title{
Acute Myocardial Infarction Followed by Cerebral Hemorrhagic Infarction in Polycythemia Vera: Case Report and Literature Review
}

\author{
XiangSen Shao ${ }^{1 \dagger}$, ZhuoTing Liu ${ }^{2 \dagger}$, ChunChang Qin ${ }^{1 *}$ and Fei Xiao ${ }^{2 *}$ \\ ${ }^{1}$ Department of Cardiology, The First Affiliated Hospital of Chongqing Medical University, Chongqing, China, ${ }^{2}$ Department of \\ Neurology, The First Affiliated Hospital of Chongqing Medical University, Chongqing, China
}

\section{OPEN ACCESS}

Edited by:

Marion Hofmann Bowman,

University of Michigan, United States

Reviewed by:

Andrew James Murphy,

Baker Heart and Diabetes

Institute, Australia

Mehmet Ozaydin,

Akdeniz Sifa Hastanesi, Turkey

Jinwei Tian,

The Second Affiliated Hospital of Harbin Medical University, China

*Correspondence:

ChunChang Qin 269363675@qq.com Fei Xiao

feixiao81@126.com

†These authors have contributed equally to this work and share first authorship

Specialty section: This article was submitted to Atherosclerosis and Vascular Medicine,

a section of the journa Frontiers in Cardiovascular Medicine

Received: 01 February 2021 Accepted: 12 August 2021

Published: 30 August 2021

Citation:

Shao X, Liu Z, Qin C and Xiao F (2021) Acute Myocardial Infarction Followed by Cerebral Hemorrhagic Infarction in Polycythemia Vera: Case Report and Literature Review.

Front. Cardiovasc. Med. 8:660999. doi: 10.3389/fcrm.2021.660999
A 60-year-old man presented to our emergency room with severe chest pain. Based on the electrocardiogram and elevated serum troponin T levels, acute coronary syndrome was suspected. Coronary angiography revealed total occlusion of the middle of the left anterior descending coronary artery. However, blood cell count abnormalities were not of concern. Twelve days later, the patient developed hemorrhagic infarction in the right parieto-occipital lobe. Acute coronary syndrome and cerebral hemorrhagic infarction were primarily caused by thrombus formation due to polycythemia vera (PV), based on the presence of increased blood consistency on admission. PV was diagnosed after bone marrow biopsy and genetic testing. The patient was treated with descending cell and antiplatelet therapy. Our case highlights the importance of the urgent identification of $\mathrm{PV}$. When acute myocardial infarction occurs in patients with no significant risk factors for cardiovascular disease, blood routine abnormalities should be paid close attention to. If PV was diagnosed as early as possible, thrombotic and hemorrhagic complications could be prevented in the early stages.

Keywords: acute myocardial infarction, polycythemia vera, cerebral infarction, thrombus, diagnosis

\section{INTRODUCTION}

A coronary artery plaque is a common cause of acute myocardial infarction (AMI), but some patients may result from other diseases such as polycythemia vera (PV). Therefore, rapid and effective identification of the cause of patients with AMI has important clinical significance. AMI due to PV is often accompanied by blood cell count abnormalities and embolism of other organs, which may be ignored in the early stages. Definitive diagnosis usually requires bone marrow biopsy and genetic examination. Most patients with PV with early intervention have a good prognosis, but the lack of early recognition may cause irreversible accidents.

\section{CASE REPORT}

A 60-year-old man presented to the emergency department with chest pain lasting for 2 days. Electrocardiography revealed ST-segment elevation in leads V1-V4 as well as elevated levels of serum troponin $\mathrm{T}(5.68 \mathrm{ng} / \mathrm{mL}$; normal range, $<0.14 \mathrm{ng} / \mathrm{mL})$ and a creatine kinase-myocardial band ( $33.73 \mathrm{U} / \mathrm{L}$; normal range, $<25 \mathrm{U} / \mathrm{L})$, indicating AMI. Routine blood tests showed a mildly increased erythrocyte level $\left(6.05 \times 10^{9} / \mathrm{L}\right.$; normal range, $\left.3.5-9.5 \times 10^{9} / \mathrm{L}\right)$ and a significantly 
increased leukocyte level $\left(20.56 \times 10^{9} / \mathrm{L}\right.$; normal range, $3.5-9.5$ $\left.\times 10^{9} / \mathrm{L}\right)$ and platelet count $\left(643 \times 10^{9} / \mathrm{L}\right.$; normal range, $100-$ $\left.300 \times 10^{9} / \mathrm{L}\right)$. However, his hemoglobin level was normal (171 g/L; normal range, 130-175 g/L) (Table 1). Emergency coronary angiography showed total obstruction of the middle of the left anterior descending coronary artery (Figures 1A,B). Therefore, leukocyte elevation was initially considered to be caused by stress after myocardial ischemia. After receiving $300 \mathrm{mg}$ aspirin and $180 \mathrm{mg}$ ticagrelor, a stent was successfully placed, and antiplatelet therapy with oral aspirin (100 $\mathrm{mg}$ per day) and ticagrelor $(90 \mathrm{mg}$ twice daily) was administered. He had no conventional risk factors for cardiovascular disease. Twelve days later, the patient suddenly developed dizziness, diplopia, and visual distortion. Cerebral computed tomography (CT) revealed a hemorrhagic infarction in the right parieto-occipital lobe (Figure 1C). The craniocervical CT angiogram (CTA) was normal. Blood routine test indicated mildly increased levels of hematocrit (55.4\%; normal range, $40-50 \%)$, leukocytes $\left(12.12 \times 10^{9} / \mathrm{L}\right.$; normal range, 3.5-9.5 $\left.\times 10^{9} / \mathrm{L}\right)$, and hemoglobin (183 $\mathrm{g} / \mathrm{L}$; normal range, $130-$ $175 \mathrm{~g} / \mathrm{L})$ and significantly increased platelet count $\left(1,128 \times 10^{9} / \mathrm{L}\right.$; normal range, $\left.100-300 \times 10^{9} / \mathrm{L}\right)$. A blood electrolyte test showed an elevated potassium concentration $(5.9 \mathrm{mmol} / \mathrm{L}$; normal range, $3.5-5.5 \mathrm{mmol} / \mathrm{L}$ ), and intravenous calcium gluconate was used to reduce blood potassium. However, in the next few days, blood routine examinations showed significantly increased levels of erythrocytes, hemoglobin, hematocrit, and leukocyte and platelet count (Table 1). Thus, bone marrow biopsy was performed, which demonstrated the proliferation of all three cell lineages. Bone marrow aspirate smears revealed active hyperplasia of bone marrow, and the ratio of $\mathrm{G} / \mathrm{E}$ (granulocytes to erythrocytes) was 1.65 . The proportion of granulocyte was increased, accounting for $56 \%$, and granulocytes account for $34 \%$. There were $>200$ megakaryocytes. There were no common risk factors of atherosclerotic vascular diseases for the patient, including smoking, hypertension, diabetes, hyperuricemia, hyperhomocysteinemia, hyperlipidemia, and atrial fibrillation, etc.

Since hematocrit and hemoglobin levels were significantly increased and bone marrow biopsy showed proliferation of all three lineage cells, PV was strongly suspected. Gene testing confirmed a positive JAK2 $2^{V 617 F}$ mutation. The patient refused venous exsanguination. Patients with thrombosis aged $>60$ years should be treated with either hydroxyurea or interferon for descending cell therapy. He was then treated with hydroxyurea $(500 \mathrm{mg})$ twice daily and interferon $(30 \mu \mathrm{g})$ once daily. Since the patient was still required to take antiplatelet drugs to prevent thrombosis and myocardial and cerebral infarctions, the patient was only administered ticagrelor every $12 \mathrm{~h}$. After 3 months of treatment, his blood routine returned to normal.

\section{DISCUSSION}

In this case, the patient had few risk factors for cardiovascular disease, and he was admitted because of acute chest pain. Coronary angiography revealed focal single-vessel disease, and other arteries were patented. During a 12-day hospital stay, the patient's red leucocyte and platelet counts remained high. We initially considered that the elevated leucocyte and platelet counts resulted from a secondary reaction from AMI, but its continued increase was inconsistent with our hypothesis. Subsequently, craniocervical CT was performed because of dizziness, diplopia, and visual distortion and revealed a cerebral hemorrhagic infarction; however, the craniocervical CTA was normal. These findings suggest that thrombus, rather than atherosis, can cause both cerebral and myocardial infarctions. The results of his blood routine, bone marrow biopsy, and genetic analysis met the diagnostic criteria for PV. Therefore, the patient developed a thrombus secondary to PV.

Myocardial infarction and stroke are complications of PV (1). However, myocardial infarction and stroke induced by common risk factors could not be explained for the abnormalities of blood routine test. In this patient, stroke occurred 12 days after myocardial infarction with a larger area identified from imaging examination, which could be attributed to the craniocerebral macrovascular disease. However, CTA of the head and neck did not reveal large blood vessel occlusion, stenosis and atherosclerosis. In addition, stroke occurred after 12-day dual therapy with aspirin and clopidogrel, and as a result, the possibility of stroke caused by cerebrovascular disease risk factors has little correlation. PV may cause sequential thrombosis in multiple organs. A previous study reported the cerebral and coronary vascular occlusive disorders in the patients (2).

Characterized by clonal proliferation of abnormal hematopoietic stem cells, PV is a Philadelphia chromosomenegative chronic myeloproliferative neoplasm. PV is diagnosed when an unexplainable increase in the hemoglobin and hematocrit levels, in addition to a JAK2 gene mutation, and a decrease in the erythropoietin level are noted (3). JAK2 mutation results in the substitution of phenylalanine to valine at V617F of the JAK2 protein, further activating tyrosine kinase expression and promoting the proliferation of hematopoietic precursors (4). Common PV complications include thrombosis and bleeding.

Fagher et al. showed that the platelet count in patients with AMI was significantly lower (5). Due to thrombosis in myocardial infarction and the participation of various inflammatory factors, many platelets are consumed, which results in a decrease in the number of platelets in circulation, which often leads to platelet maturation disorders. This was inconsistent with the laboratory findings of this case.

In a study of 1,213 patients with PV, thrombosis occurred in $19 \%$ of patients, of whom $21.7 \%$ patients had coronary thrombosis (6). Meanwhile, Hosoya et al. also showed that myocardial infarction is a common complication of PV: the incidences of myocardial infarction and ischemic stroke in patients with $\mathrm{PV}$ are 0.32 and 0.53 events $/ 100$ persons per year, respectively (1). Due to myelodysplasia, most patients with PV initially show abnormal blood test values, such as thrombocytosis, which may increase the risk of arterial thrombosis (7). Therefore, the platelet count and hematocrit level of myocardial infarction secondary to PV are always higher than those of a simple one, and this cannot be explained by myocardial infarction. In addition to the aforementioned clinical findings, splenomegaly and increases in serum lactate dehydrogenase and 
TABLE 1 | Blood routine results of the patient during hospitalization.

\begin{tabular}{|c|c|c|c|c|c|}
\hline Day of admission & Erythrocytes (10 $12 / L)$ & Hemoglobin (g/L) & Hematocrit (\%) & Platelet (109/L) & Leucocytes (109/L) \\
\hline 1st & 6.05 & 171 & 52.6 & 643 & 20.56 \\
\hline 2nd & 5.96 & 171 & 52.3 & 641 & 17.00 \\
\hline 3rd & 5.09 & 150 & 45.4 & 691 & 11.63 \\
\hline 10th & 5.53 & 159 & 48.5 & 940 & 11.95 \\
\hline 12th & 6.24 & 183 & 55.4 & 1,128 & 12.12 \\
\hline 14th & 6.44 & 187 & 58.2 & 1,192 & 15.63 \\
\hline 15th & 6.80 & 196 & 61.0 & 1,315 & 15.85 \\
\hline 16th & 6.54 & 193 & 58.2 & 1,101 & 14.94 \\
\hline 17th & 6.47 & 187 & 57.6 & 1,076 & 11.94 \\
\hline 18th & 6.33 & 184 & 56.9 & 1,049 & 10.94 \\
\hline
\end{tabular}
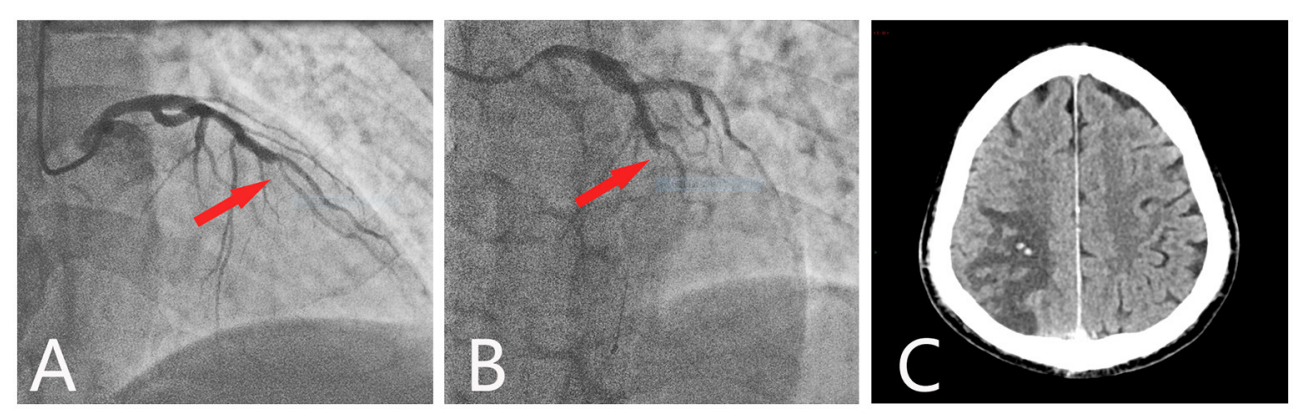

FIGURE 1 | Coronary angiography and cerebral CT. Coronary angiography showed a total occlusion at the mid-left anterior descending branch (red arrow). (A) Cranial view. (B) Left cranial view. (C) Axial cerebral CT showed hypodensity in the right parietooccipital lobe, with two spotted hyperdense areas.

neuron-specific enolase levels can be observed in patients with PV (8).

Okabe et al. (9) reported a case of myocardial infarction, even after proper stent placement, and many thromboses reformed on the stent, accompanied by significant elevation of the platelet count and hematocrit level. Bone marrow aspiration revealed bone marrow hyperplasia. This patient was clinically diagnosed with PV. Anticoagulant treatment and phlebotomy procedures were performed during hospitalization, along with the administration of hydroxyurea $(1,500 \mathrm{mg} /$ day $)$ for 1 year. Additionally, Cengiz et al. (10) retrospectively collected patients who were diagnosed with PV and presented with acute coronary syndrome. Of these, blood cell count abnormalities such as thrombocytosis were noted, considered as an inflammatory response, while some cases had missed or delayed diagnosis. They also highlighted the importance of blood cell count abnormalities in patients with AMI, especially in the absence of atherosclerotic coronary artery lesions. Although there are several case reports of PV with myocardial infarction, our patient is a rare case of myocardial infarction followed by cerebral stroke.

The exact pathogenesis of thrombosis in PV remains unclear. Some studies attribute it to large numbers of abnormal red blood cells infiltrating the arterial wall, resulting in increased blood viscosity or hyperviscosity syndrome. JAK2 mutations are present in nearly $95 \%$ of patients with $\mathrm{PV}$, and patients with JAK2 mutations have a high incidence of thrombotic events (11). Excessive activation of the JAK/STAT pathway and platelet turnover are associated with thrombosis (12). Due to accelerated platelet circulation, immature platelets are released into the blood. These compounds have a powerful hemostatic effect (12). Furthermore, enhanced platelet production of 5hydroxytryptamine, $\beta$-thromboglobulin, and platelet factor 4 due to JAK2 gene mutation also increases the risk of thrombosis (3). Compared with $J A K 2^{V 617 F}$ mutation-negative patients, positive patients have higher white blood cell and platelet counts and an increased incidence of thrombosis (13).

Hemorrhagic thrombocythemia is defined as bleeding, with platelets $>1,000 \times 10^{9} / \mathrm{L}$, which can explain the bleeding in patients with PV. When platelet counts are between 1,000 and $2,000 \times 10^{9} / \mathrm{L}$, bleeding and thrombosis may occur in response. When platelet counts are below $1,000 \times 10^{9} / \mathrm{L}$ or above $2,000 \times$ $10^{9} / \mathrm{L}$, hemorrhagic thrombocythemia can occur due to acquired von Willebrand syndrome (14). Bleeding after trauma or surgery in patients with PV is probably associated with erythrocyte fallout resulting from a high hematocrit level and impaired plateletmediated clot retraction (14). Moreover, the use of low-dose aspirin for thrombosis prevention may increase the bleeding tendency of patients with PV. Pseudohyperkalemia is also found in PV, which is associated with blood clotting and the release of potassium from platelets (15).

The treatment of PV depends on whether the patient is symptomatic or asymptomatic. The recommended drugs 
used are primarily cytoreductive and anticoagulant therapies, including hydroxycarbamide, aspirin, anagrelide, and interferonalpha (16). With the development of research, JAK inhibitors have been developed for the treatment of myeloproliferative neoplasms, including ruxolitinib and fedratinib. Recent studies by Yang et al. have suggested that selective JAK2 inhibitors could reduce the incidence of complications related to myelodysplasia by inhibiting the cell signal of hematopoietic stem and progenitor cells. Targeted drugs may eventually become the first-line therapy for patients (17). Venous exsanguination and low-dose aspirin are recommended for patients aged $<60$ years who have no thrombotic history. For patients aged $>60$ years or those with a history of thrombosis, hydroxyurea should also be administered for descending cell therapy. Interferons may also be selected.

We suggest that patients with myocardial infarction should be paid attention to the abnormal results of blood routine test, in order to avoid missed diagnosis of several rare but important causes, including PV. Blood routine and coagulation tests are two basic laboratory tests which can be carried out even in many developing countries. However, accurate diagnosis of hematological diseases requires specific hematology and molecular testing platform.

\section{DATA AVAILABILITY STATEMENT}

The original contributions presented in the study are included in the article/supplementary material, further inquiries can be directed to the corresponding author/s.

\section{REFERENCES}

1. Hosoya H, Levine JJ, Henry DH, Goldberg S. Double the trouble: acute coronary syndrome and ischemic stroke in polycythemia vera. Am J Med. (2017) 130:e237-40. doi: 10.1016/j.amjmed.2017.02.016

2. Kwaan HC, Wang J. Hyperviscosity in polycythemia vera and other red cell abnormalities. Semin Thrombosis Hemostasis. (2003) 29:4518. doi: $10.1055 /$ s-2003-44552

3. Michiels JJ, Berneman Z, Gadisseur A, Lam KH, De Raeve H, Schroyens W. Aspirin-responsive, migraine-like transient cerebral and ocular ischemic attacks and erythromelalgia in JAK2-positive essential thrombocythemia and polycythemia vera. Acta Haematol. (2015) 133:56-63. doi: 10.1159/00036 0388

4. De T, Prabhakar P, Nagaraja D, Christopher R. Janus kinase (JAK) 2 V617F mutation in Asian Indians with cerebral venous thrombosis and without overt myeloproliferative disorders. J Neurol Sci. (2012) 323:178-82. doi: 10.1016/j.jns.2012. 09.012

5. Fagher B, Sjögren A, Sjögren U. Platelet counts in myocardial infarction, angina pectoris and peripheral artery disease. Acta Medica Scand. (1985) 217:21-6. doi: 10.1111/j.0954-6820.1985.tb 01629.x

6. Gruppo Italiano Studio Policitemia. Polycythemia vera: the natural history of 1213 patients followed for 20 years. Ann Internal Med. (1995) 123:656-64.

7. Harrison CN. Platelets and thrombosis in myeloproliferative diseases. Hematology. (2005) 2005:409-15. doi: 10.1182/asheducation-2005.1.409

8. Xin $\mathrm{CH}, \mathrm{Xu}$ JQ, Sui JR, Wang XL. Analysis on 71 patients with polycythemia vera. Zhongguo Shi Yan Xue Ye Xue Za Zhi. (2012) 20:66770. https://kns.cnki.net/kcms/detail/detail.aspx?FileName=XYSY201203034\& DbName $=$ CJFQ2012

\section{ETHICS STATEMENT}

Written informed consent was obtained from the individual for the publication of any potentially identifiable images or data included in this article.

\section{AUTHOR CONTRIBUTIONS}

XS and ZL: study concept, acquisition of data and figures, and writing of the manuscript. CQ and FX: study concept and critical revision of manuscript for intellectual content. All authors cared for the patient and contributed to writing of the report.

\section{FUNDING}

This work was supported by the National Natural Science Foundation of China (No. 81541098) and Chongqing Health Commission (No. 2020MSXM113). This work was funded by the Fifth Senior Medical Talents Program of Chongqing for Yong and Middle-aged, China.

\section{ACKNOWLEDGMENTS}

We thank Dr. Xiao-Feng Lai for providing coronary artery angiograms.

9. Okabe H, Sonoda S, Abe K, Doi H, Matsumura T, Otsuji Y. Acute myocardial infarction following sequential multi-vessel occlusion in a case of polycythemia vera. $J$ Cardiol Cases. (2019) 20:111-14. doi: 10.1016/j.jccase.2019. 06.001

10. Cengiz B, Aytekin V, Bildirici U, Sahin ST, Yurdakul S, Aytekin S, et al. A rare cause of acute coronary syndromes in young adults myeloproliferative neoplasms: a case series. Rev Port Cardiol. (2019) 38:61317. doi: 10.1016/j.repc.2018.09.014

11. Levine RL, Pardanani A, Tefferi A, Gilliland DG. Role of JAK2 in the pathogenesis and therapy of myeloproliferative disorders. Nat Rev Cancer. (2007) 7:673-83. doi: 10.1038/nrc2210

12. Pedersen O, Larsen M, Kristensen S, Hvas A, Grove E. Recurrent cardiovascular events despite antiplatelet therapy in a patient with polycythemia vera and accelerated platelet turnover. Am J Case Rep. (2017) 18:945-48. doi: 10.12659/AJCR.904148

13. Lin Y, Liu E, Sun Q, Ma J, Li Q, Cao Z, et al. The prevalence of JAK2, MPL, and CALR mutations in Chinese patients with BCR-ABL1negative myeloproliferative neoplasms. Am J Clin Pathol. (2015) 144:16571. doi: 10.1309/AJCPALP51XDIXDDV

14. Michiels J, Berneman Z, Schroyens W, Van Vliet H. Pathophysiology and treatment of platelet-mediated microvascular disturbances, major thrombosis and bleeding complications in essential thrombocythaemia and polycythaemia vera. Platelets. (2004) 15:67-84. doi: 10.1080/095371003100016 46969

15. Ong YL, Deore R, El-Agnaf M. Pseudohyperkalaemia is a common finding in myeloproliferative disorders that may lead to inappropriate management of patients. Int $J$ Lab Hematol. (2010) 32:e151-7. doi: 10.1111/j.1751-553X.2008.0 1114.x 
16. Silver RT, Kiladjian JJ, Hasselbalch HC. Interferon and the treatment of polycythemia vera, essential thrombocythemia and myelofibrosis. Expert Rev Hematol. (2013) 6:49-58. doi: 10.1586/eh m.12.69

17. Tang Y, Liu W, Wang W, Fidler T, Woods B, Levine RL, et al. Inhibition of JAK2 suppresses myelopoiesis and atherosclerosis in apoe mice. Cardiovasc Drugs Therapy. (2020) 34:145-52. doi: 10.1007/s10557-020-0 6943-9

Conflict of Interest: The authors declare that the research was conducted in the absence of any commercial or financial relationships that could be construed as a potential conflict of interest.
Publisher's Note: All claims expressed in this article are solely those of the authors and do not necessarily represent those of their affiliated organizations, or those of the publisher, the editors and the reviewers. Any product that may be evaluated in this article, or claim that may be made by its manufacturer, is not guaranteed or endorsed by the publisher.

Copyright (C) 2021 Shao, Liu, Qin and Xiao. This is an open-access article distributed under the terms of the Creative Commons Attribution License (CC BY). The use, distribution or reproduction in other forums is permitted, provided the original author(s) and the copyright owner(s) are credited and that the original publication in this journal is cited, in accordance with accepted academic practice. No use, distribution or reproduction is permitted which does not comply with these terms. 